\title{
Magnetic anchor-guided endoscopic submucosal dissection for colonic tumor
}
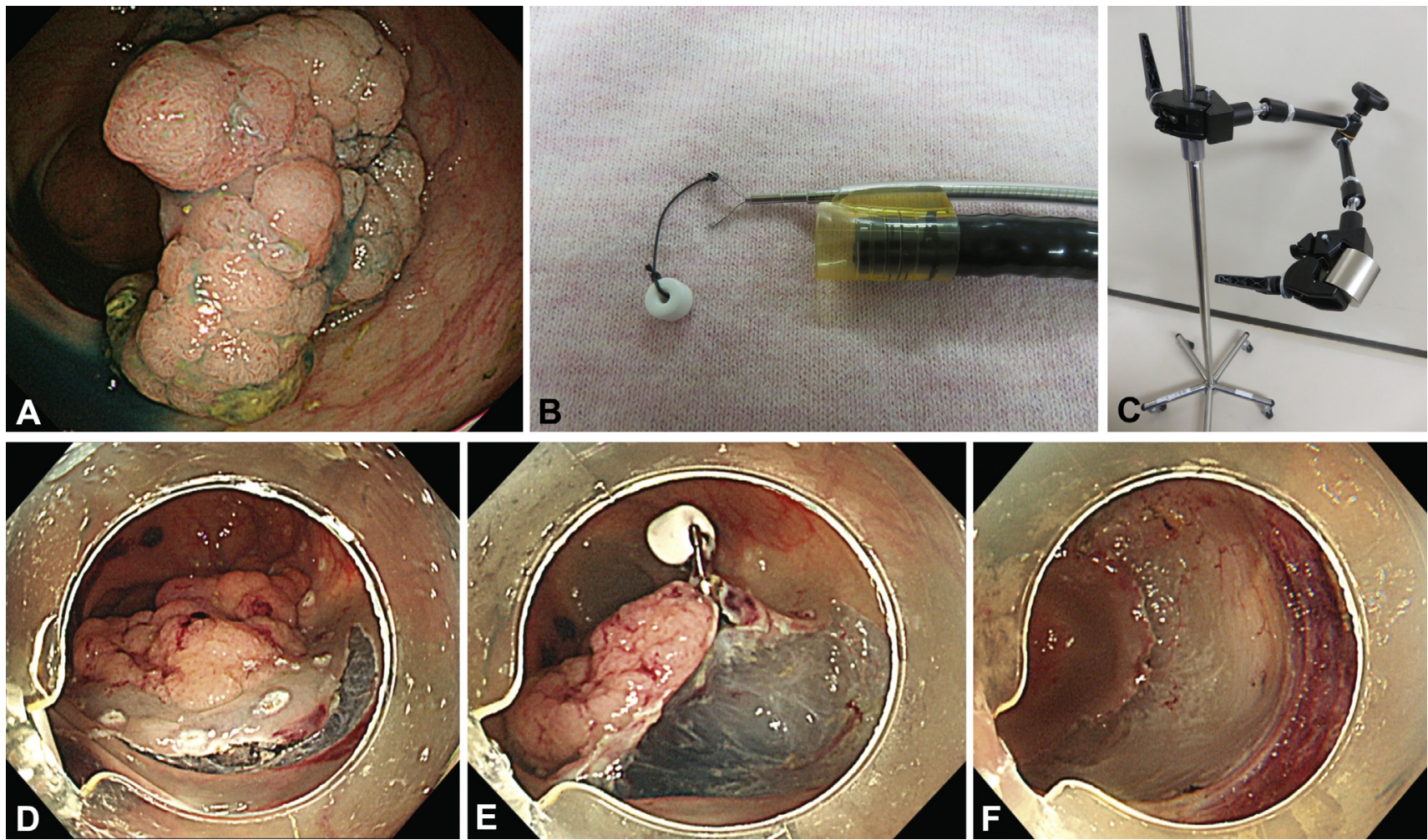

Figure 1. Magnetic anchor-guided endoscopic submucosal dissection for a laterally spreading tumor. A, Endoscopic image of the lesion in the cecum. B, The magnet attached to a hemoclip with thread. $\mathbf{C}$, The external magnet with a flexible arm. D, After partial dissection, the mucosal edge before magnetic anchor is attached. E, Direct visualization of the submucosal layer by traction using magnetic anchor. F, Endoscopic view after endoscopic submucosal dissection, showing a large artificial ulcer.

Colorectal endoscopic submucosal dissection (ESD) is technically difficult and is highly associated with a risk of perforation because of the thin muscle layer and poor maneuverability of the endoscope. Magnetic anchorguided ESD (MAG-ESD) with use of a large external electromagnet provides adequate direction and degrees of traction in various locations. For application in daily clinical practice, we have previously reported the use of MAG-ESD with a neodymium magnet in live dog stomachs. This case report describes colonic MAG-ESD with the use of this permanent magnet.

An 83-year-old woman was referred to our hospital for a laterally spreading tumor, granular type, in the cecum (Fig. 1A). After a partial mucosal cut was made by a Flush knife BT (Fujifilm Co, Tokyo, Japan), a magnetic anchor consisting of an internal neodymium magnet attached to a hemoclip (Zeoclip; Zeon Medical Inc, Tokyo, Japan) was attached to the proximal mucosal edge of the lesion. A transparent hood fitted with a mucosal forceps channel (A-CS; TOP Co, Tokyo, Japan) was used for smooth delivery of the magnetic anchor (Fig. 1B). The external neodymium magnet was locked by a flexible arm (FA-MVC2; SFC Co Ltd, Kanagawa, Japan) to keep it in position during ESD (Fig. 1C). Adequate countertraction with good visualization was obtained by use of the external magnet, and submucosal dissection was performed (Figs. 1D and E). MAG-ESD with neodymium magnets was successful (Fig. 1F) (Video 1, available online at www.VideoGIE.org). The patient was discharged without adverse events. Histopathologic evaluation of the lesion 
showed tubular adenocarcinoma with a free surgical margin and with shallow submucosa.

We hope that this MAG system will facilitate all difficult ESD procedures.

\section{DISCLOSURE}

All authors disclosed no financial relationships relevant to this publication.
Ippei Matsuzaki, MD, PhD, Sho Isobe, MD, Ken Hirose, MD, Takahiro Marukawa, MD, Masaya Esaki, MD, Department of Gastroenterology, Yamashita Hospital, Ichinomiya, Japan

Copyright (c) 2017 American Society for Gastrointestinal Endoscopy. Published by Elsevier Inc. This is an open access article under the CC BY-NC-ND license (http://creativecommons.org/licenses/by-nc-nd/4.0/).

http://dx.doi.org/10.1016/j.vgie.2017.01.016 